\title{
Relación de la hipoacusia con el ausentismo laboral en la Planta MOLICAL S.A.C - 2017
}

\author{
Relationship of hearing loss with absenteeism at the \\ MOLICAL S.A.C Planta - 2017
}

Esteve Alan Gonzales Ortiz ${ }^{1}$

Recibido: 23/09/2020 - Aprobado: 05/07/2021 - Publicado: 23/12/2021

\begin{abstract}
RESUMEN
La presente investigación tiene como principal objetivo determinar si existe relación entre la hipoacusia con el ausentismo laboral. Esta investigación utilizó como metodología el tipo de investigación observacional, descriptivo transversal de carácter retrospectivo. De acuerdo a la profundidad, es un estudio correlacional. La población de estudio comprendió el 100\% de trabajadores de la planta MOLICAL S.A.C, lo que correspondió a 87 trabajadores. Luego del análisis estadístico se determinó que el valor de significación observada es de $p=0.004$, por lo que vemos es superior a 7,815, por consecuencia, la hipótesis nula es descartada y la hipótesis de la investigación es admitida, debido a que se encuentra evidencia necesaria para afirmar que existe una relación, lo cual nos permite asentir que las Hipoacusia incide en el Ausentismo de los trabajadores (Rojas Concha, 2014). Estos resultados en la investigación indican que se presenta evidencia suficiente para aceptar la afirmación de que existe relación entre la hipoacusia y el ausentismo laboral en la Planta MOLICAL S.A.C, ya que el 66,1\% de los trabajadores de la planta se ven afectados negativamente por hipoacusia, además se pudo evidenciar que del total de trabajadores que arrastraron alguna problemática de salud durante el año 2017, el 21,8\% de trabajadores presentaron problemas del tipo auditivo. Asimismo se pudo establecer que el alto índice de ausentismo laboral afecta negativamente en un $72,5 \%$ a las actividades realizadas en la empresa mencionada. Por estos motivos en la Planta de producción de MOLICAL S.A.C y otras plantas similares seria de suma importancia implementar medidas preventivas referidas a la afectación de la hipoacusia laboral y el monitoreo de ausencias justificas y no justificadas.
\end{abstract}

Palabras claves: Hipoacusia; ausentismo; salud; afectación; prevención.

\begin{abstract}
The main objective of this research is to determine if there is a relationship between hearing loss and absenteeism from work. This research used as a methodology the type of observational, descriptive, cross-sectional, retrospective research. According to the depth, it is a correlational study. The study population comprised $100 \%$ of workers at the MOLICAL S.A.C plant, which corresponded to 87 workers. After the statistical analysis, it was determined that the observed significance value is $p=0.004$, so we see it is higher than 7.815 , consequently, the null hypothesis is discarded and the research hypothesis is admitted, because evidence is found necessary to affirm that there is a relationship, which allows us to agree that hearing loss affects absenteeism of workers (Rojas Concha, 2014). These research results indicate that sufficient evidence is presented to accept the statement that there is a relationship between hearing loss and absenteeism from work at the MOLICAL S.A.C Plant, since $66.1 \%$ of the plant workers are negatively affected by hearing loss, it was also possible to show that of the total number of workers who suffered a health problem during the year $2017,21.8 \%$ of workers presented health problems. Auditory type. Likewise, it was established that the high rate of absenteeism negatively affects $72.5 \%$ the activities carried out in the aforementioned company. For these reasons, in the MOLICAL S.A.C production plant and other similar plants, it would be extremely important to implement preventive measures related to the impact of occupational hearing loss and the monitoring of justified and unjustified absences.
\end{abstract}

Keywords: Hearing loss; Absenteeism; Health; affectation; prevention.

\footnotetext{
1 MOLICAL S.A.C, Av. Gerardo Unger N 5951 Los Olivos, Lima, Perú.

E-mail: egonzales@molical.com - ORCID: https://orcid.org/0000-0002-4711-2515
} 


\section{INTRODUCCIÓN}

El ausentismo laboral está considerado como un suceso de relevancia para las empresas e instituciones de todo el mundo, a lo largo de los años se ha tomado realce a las estadísticas sobre las horas trabajadas, el ausentismo laboral ha ganado importancia en este entorno, pues las ausencias en el trabajo son un fenómeno que resulta considerablemente costoso para las organizaciones. Según la (OIT, 2010) en el mundo aproximadamente se pierde hasta el $4 \%$ del producto bruto Interno por el costo que generan las ausencias laborales.

La Hipoacusia inducida por ruido está considerada entre las enfermedades ocupacionales más relevantes por la Organización Internacional del Trabajo (OIT) y ocurre predominantemente en trabajadores con edades superiores a los 40 años. El ruido es una problemática de salud pública que considera de 1 a 300 millones de personas afectadas en la alteración de su sistema auditivo. Los trabajadores expuestos al ruido ocupacional son afectados entre el $7 \mathrm{y}$ $21 \%$ por pérdida auditiva en el mundo, el porcentaje más bajo se presenta en los países industrializados, donde la repercusión aminora y el porcentaje más alto se presenta en los países en vías de desarrollo. (Carpio \& Alvares, 2017)

El Perú no es ajeno a esta problemática, aunque no hay muchos estudios con respecto a las causas del ausentismo en el país. El Estado peruano, de acuerdo con sus protocolos de exámenes médicos ocupacionales y guías de diagnóstico de los exámenes médicos obligatorios por actividad, tiene por finalidad "Proteger y promover la seguridad y salud de los trabajadores, así como generar ambientes de trabajo saludables" (Allpas Gómez et al., 2016).

MOLICAL S.A.C, es una empresa del sector industrial, que inicia sus actividades en el año 2001 y como actividad principal desarrolla la pulverización y micronización de minerales no metálicos, para su comercialización, teniendo como materias primas, bentonita, caolín, carbonato de calcio, arcillas y oxido de calcio. La pulverización de la materia prima es realizada por molinos de bolas y de martillo, además de las actividades de pulverización en la planta se desarrollan actividades de almacenamiento, mantenimiento, carga y descarga, así como actividades administrativas.

En la planta de producción de la empresa MOLICAL S.A.C, hoy en día el ausentismo laboral ha dado indicios de ser causados en mayor cantidad debido a problemas de hipoacusia por lo cual se ha convertido en un dolor de cabeza, el ausentismo de empleados genera costos adicionales para la organización, para reducir las ausencias causadas por los casos de hipoacusia, es necesario que los trabajadores puedan identificar los síntomas de la hipoacusia y de las consecuencias que esta enfermedad ocupacional pueda ocasionar, para que de esta manera sea el punto de partida para que los trabajadores asuman el compromiso de cumplir con las medidas de planificación y las decisiones que adopte la gerencia de la empresa para hacer frente al problema.

El tipo de lesión auditiva principal se da por la exposición a ruidos fuertes lo cual genera pérdida auditiva ya que en la planta MOLICAL S.A.C, los trabajadores están expuestos a ruidos fuertes generados principalmente por los molinos de bolas y de martillo, por periodos largos de tiempo, a esta pérdida auditiva también se le denomina pérdida auditiva inducida por el ruido.

Teniendo en cuenta lo expuesto en los párrafos anteriores el presente estudio tuvo como objetivo: determinar la relación entre la hipoacusia con el ausentismo laboral en los trabajadores de la planta MOLICAL S.A.C - 2017, con lo cual este estudio abre un camino amplio para que, desde la gerencia general de la empresa y los tomadores de decisiones, apoyados en las normativas internas se definan indicadores que identifiquen posibles casos de hipoacusia, además de otras patologías y su relación con el ausentismo laboral en general, en todos los trabajadores de la planta, para que de esta manera a nivel de gerencia se pueda planificar, prevenir y corregir los caos de ausentismo laboral y conocer su impacto económico.

\section{MÉTODOS}

La investigación desarrolla un tipo de estudio observacional, descriptivo transversal con carácter retrospectivo. Es decir, la investigación es no experimental porque se observan los fenómenos tal y como se dan en su contexto natural y no hay manipulación de las variables.

La población de estudio estuvo constituido por 87 trabajadores que es el total del personal de trabajadores de la Planta MOLICAL-Lima, distribuidos en las áreas de molienda, mantenimiento, almacén, supervisores, auxiliares, jefes y demás personal administrativo que labora en la mencionada planta, y que son susceptibles a las ausencias del trabajo relacionadas o no con la hipoacusia y que se encontraban en nómina en la organización en el año 2017, incluyendo al personal que ingresó a laborar como trabajadores nuevos y personal que renuncio en este periodo de tiempo en la organización.

Para el presente trabajo se tomó como muestra al total de la población que viene a ser el total de trabajadores que laboraron en la planta MOLICAL S.A.C y que fueron encuestados en el año 2017, el total de trabajadores lo integran todos los trabajadores de la planta distribuidos en las siguientes áreas: molienda, mantenimiento, volantes, almacén, supervisores, auxiliares, jefes y personal de las áreas administrativas de la empresa.

Para la recolección de la información se utilizó una recopilación de datos sobre el ausentismo laboral y los casos de hipoacusia en el personal de la planta MOLICAL S.A.C, durante el año 2017.

En la etapa de recolección de información y datos se manejó el uso las siguientes fuentes:

- Información disponible de la empresa (archivos)

- Base de datos de la Oficina de Recursos Humanos

- Monitoreos de ruido

- Registros de asistencia

- Hojas de vida 
- Exámenes médicos

- Cuestionarios

\section{RESULTADOS}

3.1. Ausentismo en la planta MOLICAL S.A.C.

Revisando las hojas de asistencia a la planta MOLICAL S.A.C, así como los reportes de la Oficina de Recursos Humanos, se pudo verificar que en el año 2017 se presentaron 341 días de ausentismo laboral de los cuales 212 fueron justificados lo que representa un $62 \%$ de ausencias justificadas y 129 no fueron justificadas, lo que representa un $38 \%$ de ausencias no justificadas, el número de personas que presentaron ausentismo durante el año 2017 fue de 47 (Figura 1).

\subsection{Análisis y resultados de la variable hipoacusia}

El estudio analizó los porcentajes de niveles de hipoacusia, clasificándolos en hipoacusia normal, Hipoacusia leve, Hipoacusia moderada e Hipoacusia severa, en la Tabla 1, se puede observar que 27 trabajadores sufren de hipoacusia severa lo que se traduce en un 31\% del total, 26 trabajadores sufren de hipoacusia moderada, lo que refleja el 29.9\%, 18 trabajadores sufren de hipoacusia leve, lo que implica que el $20.7 \%$ de trabajadores tendrían hipoacusia leve, además 16 trabajadores tienen hipoacusia normal, lo que significa que el $18.4 \%$ del total de trabajadores no tienen problemas de hipoacusia (Figura 2).

\section{Tabla 1. Hipoacusia}

\begin{tabular}{lcc}
\hline & Frecuencia & Porcentaje \\
\hline Hipoacusia Normal & 16 & 18.4 \\
Hipoacusia Leve & 18 & 20.7 \\
Hipoacusia Moderada & 26 & 29.9 \\
Hipoacusia Severa & 27 & 31.0 \\
\hline Total & $\mathbf{8 7}$ & $\mathbf{1 0 0 . 0}$ \\
\hline
\end{tabular}

3.3. Análisis y resultados de la variable ausentismo

En el estudio también se analizó la variable ausentismo, analizando los porcentajes de ausentismo justificado y ausentismo injustificado en la planta MOLICAL S.A.C, del año 2017, teniendo como resultado los datos expresados

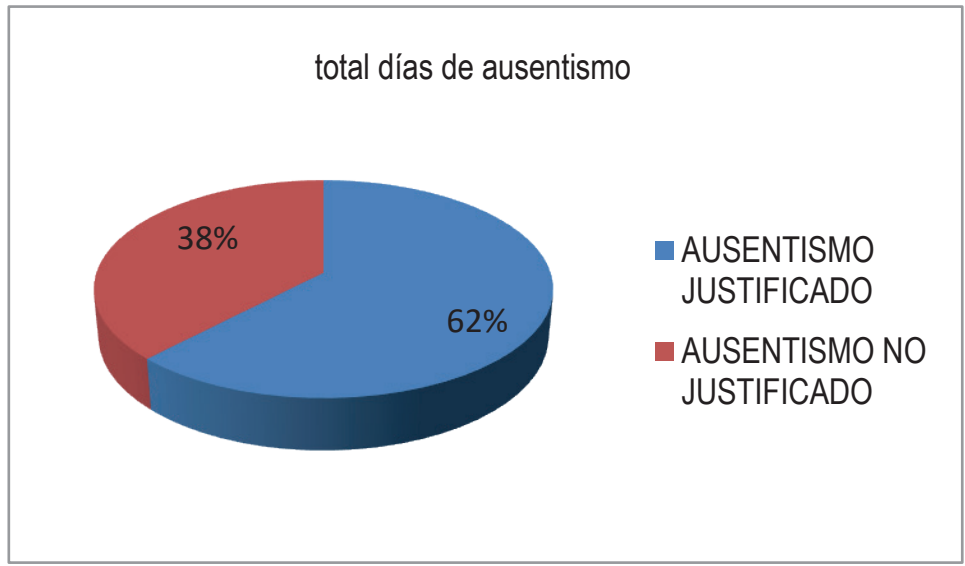

Figura 1. Total ausentismo

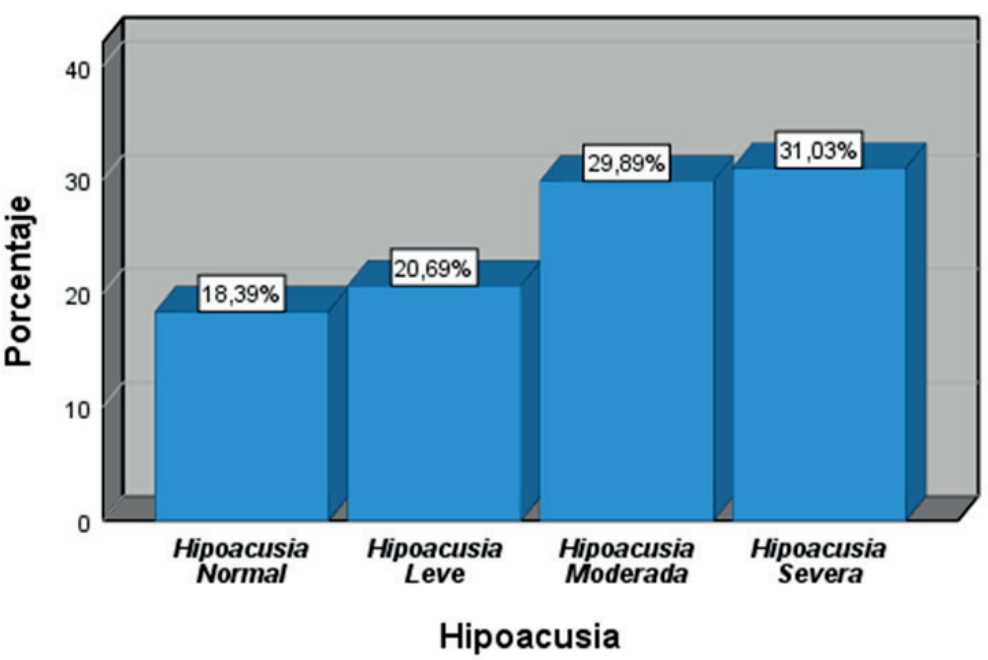

Figura 2. Niveles de Hipoacusia 
en la Tabla 2, donde indica que el $62.1 \%$ de ausentismo es justificado en la planta, y un $37.9 \%$ de ausentismo no ha podido ser justificado, ni existe evidencia para poder determinar sus causas (Figura 3 ).

Tabla 2. Ausentismo

\begin{tabular}{lcc}
\hline & Frecuencia & Porcentaje \\
\hline Ausentismo Justificado & 54 & 62.1 \\
Ausentismo Injustificado & 33 & 37.9 \\
\hline Total & $\mathbf{8 7}$ & $\mathbf{1 0 0 . 0}$ \\
\hline
\end{tabular}

\subsection{Análisis y resultados de la salud de los trabajadores}

Estos resultados nos muestran que los problemas auditivos tienen un porcentaje considerable de afecciones a la salud de los trabajadores ya que está representada por un $21.8 \%$, comparado a los otros problemas de salud que se representa por un $78.2 \%$, tal como se verifica en la Tabla 3 (Figura 4).

Tabla 3. Salud de los Trabajadores

\begin{tabular}{lcc}
\hline & Frecuencia & Porcentaje \\
\hline Problemas Auditivos & 19 & 21.8 \\
Otros Problemas de Salud & 68 & 78.2 \\
\hline Total & $\mathbf{8 7}$ & $\mathbf{1 0 0 . 0}$ \\
\hline
\end{tabular}

\subsection{Análisis de las actividades de los trabajadores}

En la Tabla 4, se muestra el porcentaje de los trabajadores que pertenecen a determinada área y actividades de trabajo, mostrando que el $28.7 \%$ de trabajadores que pertenecen al área de molienda o pulverización, el $25.3 \%$ pertenecen al área de almacén y despacho, el $21.8 \%$ desarrollan sus actividades en el área de mantenimiento, el $17.2 \%$ pertenecen al área de carga y descarga y el $6.9 \%$ desarrollan actividades administrativas y/o de oficina (Figura 5).

Tabla 4. Actividades de los trabajadores

\begin{tabular}{lcc}
\hline & Frecuencia & Porcentaje \\
\hline Actividad Pulverización & 25 & 28.7 \\
Actividad Almacenamiento & 22 & 25.3 \\
Actividad Mantenimiento & 19 & 21.8 \\
Actividad Carga y Descarga & 15 & 17.2 \\
Actividad Administrativa & 6 & 6.9 \\
\hline Total & 87 & 100.0
\end{tabular}

\subsection{Prueba de Hipótesis}

Es el proceso de comprobación del conocimiento científico del modelo teórico-empírico que fue corroborado a partir de los datos recopilados y que mediante un modelo estadístico determinó la aceptación o rechazo de la hipótesis de la investigación.

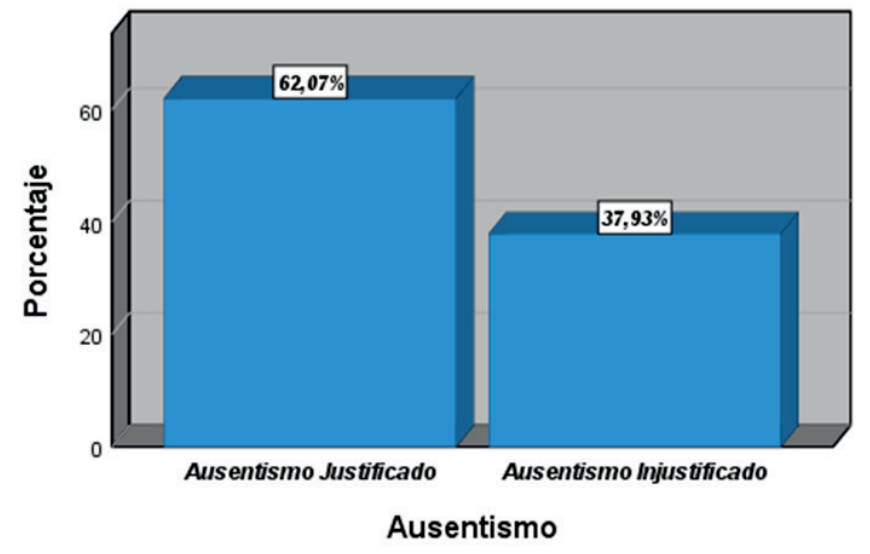

Figura 3. Porcentaje de Ausentismo

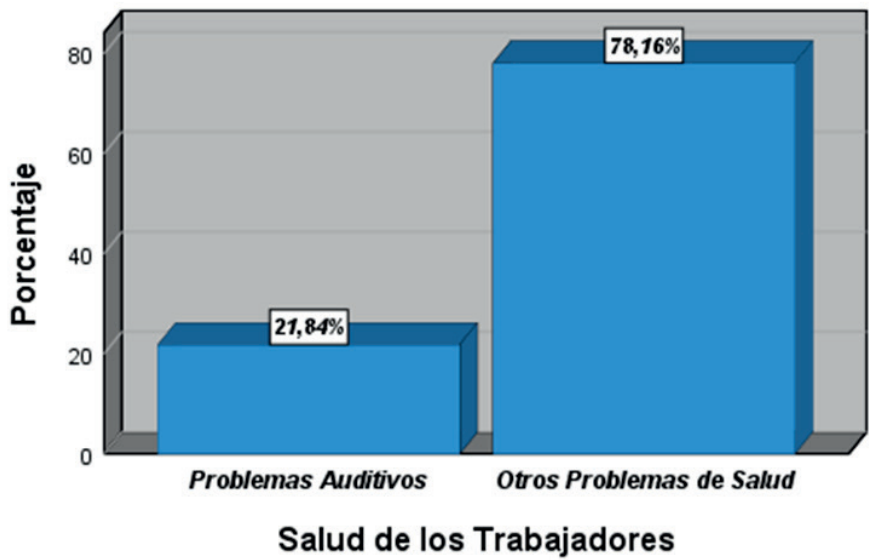

Figura 4. Problemas de Salud de los Trabajadores 
En el caso de la presente investigación transversal de carácter retrospectivo, se utilizó la estadística paramétrica usando la prueba de correlación de Pearson (Amat, 2016).

Como las variables y las dimensiones son no normales se aplicó la estadística paramétrica usando prueba Chi cuadrado y Correlación de Pearson.

Hipótesis General: La Hipoacusia está relacionada con los casos de ausentismo de los trabajadores de la Planta MOLICAL S.A.C

Ho: La hipoacusia no está relacionada con los casos de ausentismo de los trabajadores de la Planta MOLICAL S.A.C

H1: Las Hipoacusia está relacionada con los casos de ausentismo de los trabajadores de la Planta MOLICAL S.A.C. (Tabla 5, Figura 6).
Tabla 5. Hipoacusia*Ausentismo

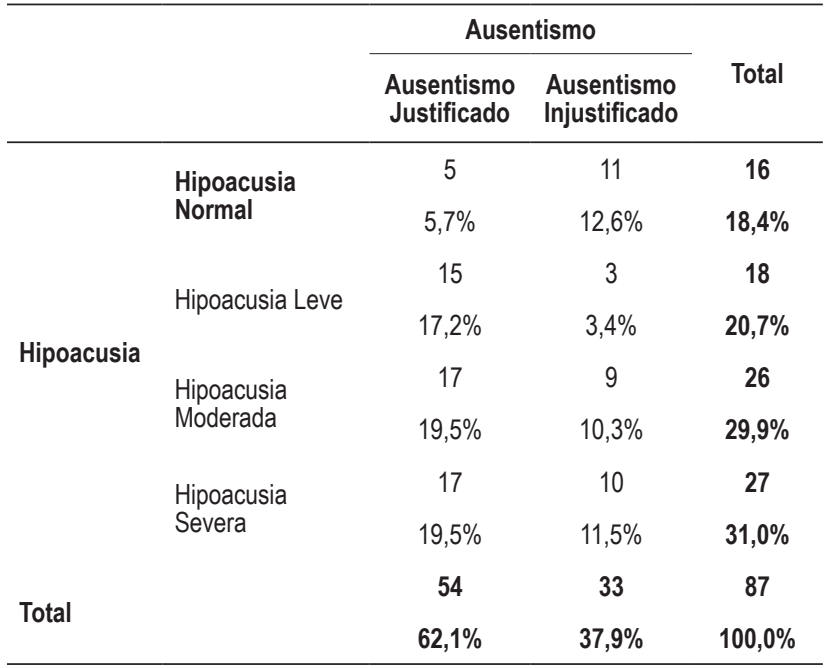

Fuente: Trabajadores de la Planta MOLICAL S.A.C.

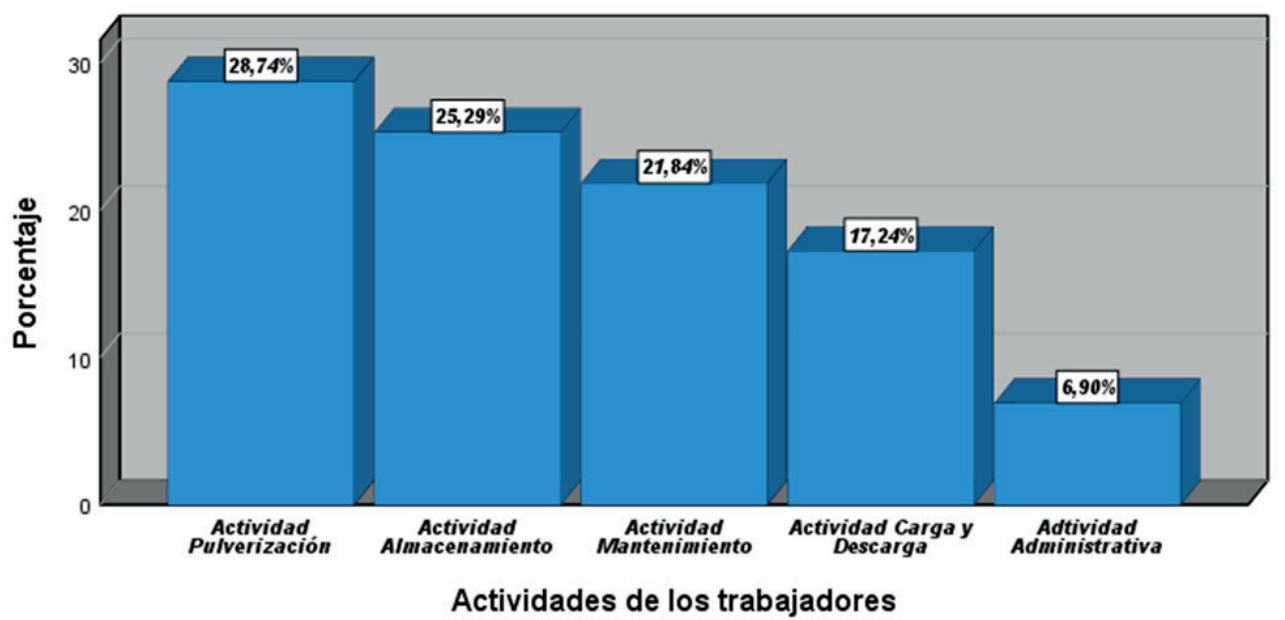

Figura 5. Actividades de los Trabajadores

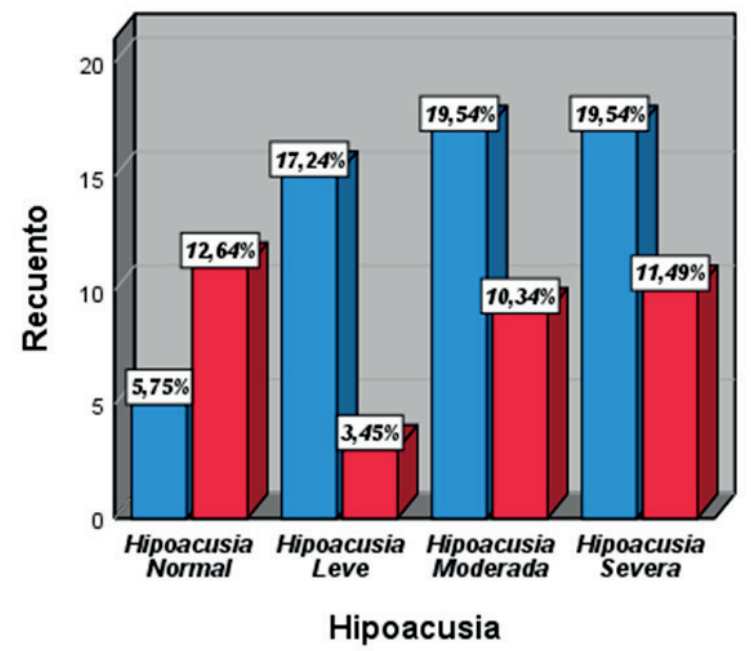

\section{Ausentismo}

$\square$ Ausentismo Justificado

Ausentismo

Injustificado

Figura 6. Comparación del ausentismo justificado con el ausentismo injustificado 


$$
\mathrm{X}^{2}=10.043 \quad \alpha=0.018 \mathrm{p}=0.05
$$

Regla de Decisión:

Si $\alpha=5 \%$, con (R-1) (S-1) = (4-1) (2-1) = $3 \mathrm{gl}$.

X2 0.05, $3=7,815$; la regla de decisión es:

"No Rechazar la hipótesis nula si” X20.05,3 < 7,815

Si Rechazar la hipótesis nula si” X2 0.05, $3>7,815$

A partir de la prueba de Chi cuadrada de la investigación se obtuvo que el X2 $=10.043 \mathrm{GL}=3$ y $\mathrm{p}=0.004$, por lo que resulta que la Chi cuadrada de la investigación es mayor a 7,815, por consecuente, se rechaza la hipótesis nula y se acepta la hipótesis de investigación, dado que existe suficiente evidencia para asumir que existe una relación, lo que nos permite afirmar que las Hipoacusia incide en el Ausentismo de los trabajadores (Figura 7, Tabla 6).

Tabla 6. Medidas simétricas

\begin{tabular}{lccc}
\hline & Valor & $\begin{array}{c}\text { Significación } \\
\text { aproximada }\end{array}$ \\
\hline $\begin{array}{l}\text { Nominal por } \\
\text { Nominal }\end{array}$ & $\begin{array}{l}\text { Coeficiente de } \\
\text { contingencia }\end{array}$ & 0.652 & 0.018 \\
\hline $\mathrm{N}^{\circ}$ de casos válidos & 52 & \\
\hline
\end{tabular}

La influencia entre la hipoacusia y el ausentismo del trabajador presenta el valor de 0.652 lo que significa que existe una influencia significativa alta.

En cuanto a la hipótesis especifica 01 . El nivel alto de hipoacusia afecta negativamente la salud de los trabajadores de la planta MOLICAL S.A.C.

H0: El nivel alto de hipoacusia no afecta negativamente la salud de los trabajadores de la planta MOLICAL S.A.C.
H1: El nivel alto de hipoacusia afecta negativamente la salud de los trabajadores de la planta MOLICAL S.A.C.

El resultado sobre nivel de significación teórica es $\alpha=$ 0.05 , que pertenece al nivel de confiabilidad del $95 \%$.

\section{Función de Prueba}

Se desarrolló a partir de la prueba no paramétrica correspondiente a la prueba Pearson (ver tabla 7).

\section{$\underline{\text { Regla de decisión }}$}

Cuando la significación observada "p" de los coeficientes del modelo logístico es menor que $\alpha$ entonces se rechaza la $\mathrm{H} 0$.

Cuando la significación observada "p" de los coeficientes del modelo logístico es mayor que $\alpha$, entonces no se rechaza la $\mathrm{H} 0$.

\section{Cálculos}

Tabla 7. Prueba de Rho de Spearman Correlaciones

\begin{tabular}{lllc}
\hline & & $\begin{array}{c}\text { Salud de los } \\
\text { Trabajadores }\end{array}$ \\
\hline \multirow{4}{*}{ Pearson } & Coeficiente de Pearson &,$- 661^{*}$ \\
& $\begin{array}{l}\text { Nivel Alto de } \\
\text { Hipoacusia }\end{array}$ & Sig. (bilateral) & 0,001 \\
& $n$ & 87
\end{tabular}

*. La correlación es significativa en el nivel 0,05 (bilateral)

Como se observa de la Tabla 8, existen diferencias significativas por lo cual el nivel alto de hipoacusia afecta negativamente la salud

Por lo tanto, se acepta la Hipótesis especifica 01 de investigación.

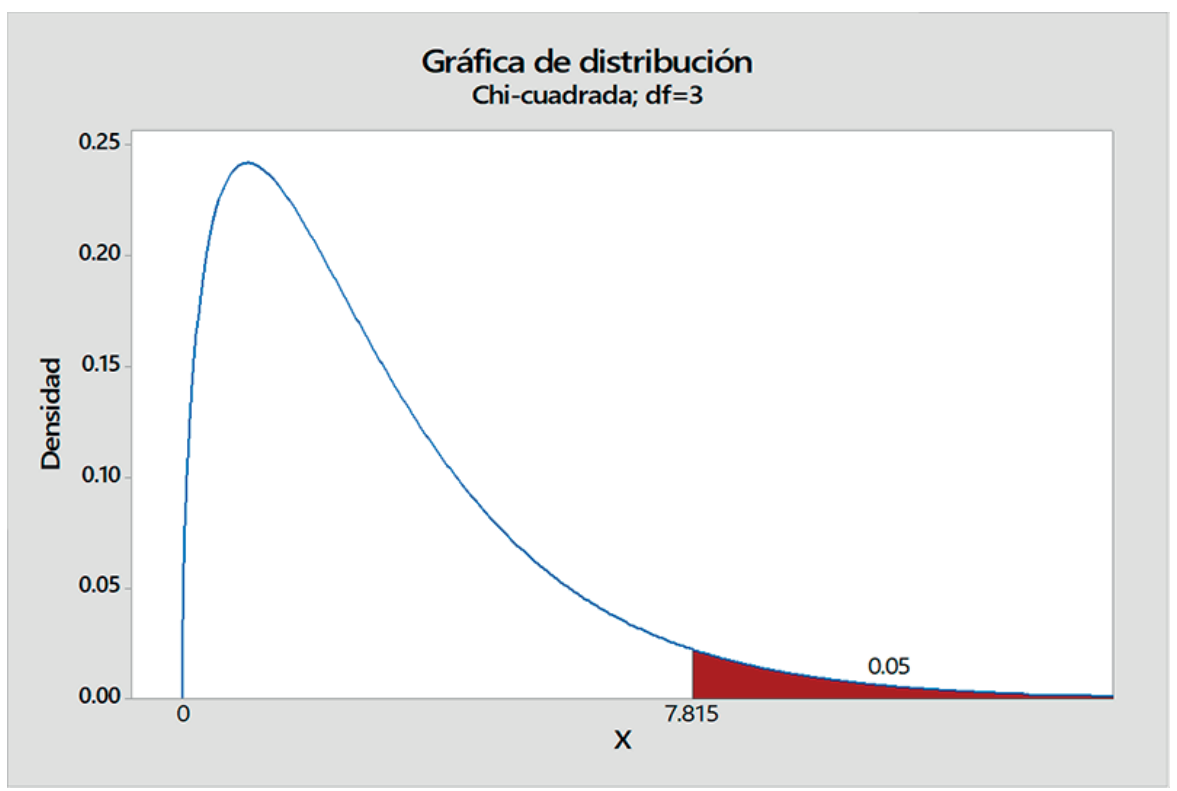

Figura 7. Distribución de Prueba Chi Cuadrado para la Hipótesis 
El estudio permite inferir también que en la Planta Industrial de MOLICAL S.A.C se presentan casos considerables de problemas de hipoacusia en diferentes niveles y que los niveles altos de hipoacusia afectan de manera negativa la salud de los trabajadores de la planta MOLICAL S.A.C.

Se ha podido determinar además en este estudio que el 18,39\% presentan una hipoacusia normal, el 20,69\% una hipoacusia leve, el 29,89\% una hipoacusia moderada y el $31.03 \%$ una hipoacusia severa.

El estudio también nos permitió determinar que el alto índice de ausentismo laboral afecta negativamente el desarrollo de las actividades de los trabajadores en un $75.2 \%$ en la Planta de MOLICAL S.A.C.

En cuanto a la hipótesis Especifica 02. El alto índice de ausentismo laboral afecta negativamente las actividades de los trabajadores de la Planta MOLICAL S.A.C.

H0: El alto índice de ausentismo laboral no afecta negativamente las actividades de los trabajadores de la Planta MOLICAL S.A.C.

H1: El alto índice de ausentismo laboral afecta negativamente las actividades de los trabajadores de la Planta MOLICAL S.A.C.

El nivel de significación teórica es $\alpha=0.05$, que pertenece al nivel de confiabilidad del $95 \%$.

\section{Función de Prueba}

Se desarrolló a partir de la prueba no paramétrica utilizando la prueba de correlación de Pearson.

\section{$\underline{\text { Regla de decisión }}$}

Cuando la significación observada "p" de los coeficientes del modelo logístico es menor que $\alpha$ entonces se rechaza la $\mathrm{H} 0$.

Cuando la significación observada "p" de los coeficientes del modelo logístico es mayor que $\alpha$, entonces no se rechaza la $\mathrm{H} 0$.

\section{Cálculos}

Tabla 8. Prueba de Rho de Spearman Correlaciones

\begin{tabular}{lllc}
\hline & & $\begin{array}{c}\text { Actividades de } \\
\text { los Trabajadores }\end{array}$ \\
\hline \multirow{2}{*}{ Pearson } & $\begin{array}{l}\text { Alto Indice de } \\
\text { Ausentismo } \\
\text { Laboral }\end{array}$ & Coeficiente de Pearson &,$- 752^{*}$ \\
& Sig. (bilateral) & 0,004 \\
$\mathrm{n}$ & $\mathrm{n}$ & 87 \\
\hline
\end{tabular}

*. La correlación es significativa en el nivel 0,05 (bilateral).

Como se observa de la Tabla 8, existen diferencias significativas por lo cual el alto índice de ausentismo laboral afecta negativamente las actividades

Dado que el valor de significación observada $\mathrm{p}=$ 0.004 es menor al valor de significación teórica $\alpha=0.05$, se rechaza la Hipótesis nula. Por ello se concluye que existe un alto índice de ausentismo laboral que afecta negativamente las actividades de los trabajadores en un 75.2\% (correlación negativa) de la Planta MOLICAL S.A.C.

Por lo tanto, se acepta la Hipótesis específica 02 de investigación.

\section{DISCUSIÓN}

No se han encontrado investigaciones sobre el ausentismo relacionado por casos de hipoacusia sin embargo, podemos comparar nuestros resultados con algunas investigaciones sobre el ausentismo como describimos a continuación:

Como antecedentes del presente estudio, se pudo encontrar información sobre los problemas de hipoacusia, siendo estos problemas los que desde hace años afectan a los trabajadores de las industrias especialmente a la industria minera es así que en el libro Factores de Riesgo Laboral en Minería (Rodríguez García, 2014), se menciona que en la minería de acuerdo al INVEPROMI (instituto de Investigación de Enfermedades Profesionales Mineras) a principios de los años de 1983 a 1993, existía un 22.5\% de neumoconiosis, con un $20 \%$ complicados con tuberculosis pulmonar, el $55 \%$ de 810 trabajadores expuesto a ruido tenían hipoacusia neurosensorial (trauma acústico, problema auditivo de origen ocupacional)

Asimismo, se halló en la investigación titulada Análisis del ausentismo laboral por enfermedad en el personal de SEDAPAL, en el periodo 2001-2013, que de los 136873 días de ausentismo laboral estos eran ocasionados por enfermedad. Los descansos médicos de 1 a 3 días de duración tuvieron como principales causas las enfermedades del aparato digestivo, del aparato respiratorio y del aparato locomotor. En los casos de los descansos médicos con más de tres días de duración, las principales causas fueron traumatismos, enfermedades del aparato locomotor y del aparato digestivo (Rodríguez García, 2014).

En el caso del estudio realizado en la planta MOLICAL S.A.C, se obtuvo 35 ausencias justificadas por accidentes laborales, 122 ausencias justificadas por enfermedades comunes, 53 por casos de maternidad, y 2 por otras causas personales, 129 ausencias no fueron justificadas, dentro de las cuales se encontrarían también las ausencias por problemas de hipoacusia.

A partir de los resultados de la investigación, se puede afirmar que esta aporta un beneficio para la industria en general, debido a que es la base para diseñar herramientas para reestructurar de forma efectiva el actual sistema de gestión de seguridad y salud en el trabajo, el cual contemple las medidas para prevenir, mitigar y controlar los casos de ausentismo laboral especialmente los que está relacionado con los problemas de pérdida auditiva o hipoacusia.

Por otra parte, busca alertar a la gerencia de la empresa y la industria en general sobre la relación hallada entre el ausentismo laboral presentado en la empresa con los casos de hipoacusia, para que a través de la toma de 
decisiones, se ejecute las medidas efectivas para prevenir y mitigar los casos de hipoacusia laboral que a la vez son causantes de un porcentaje de ausentismo y posteriormente reflejan frenos en los tiempos de entrega de los productos y en ocasiones se llega a paralizar la propia producción, en periodos considerables de tiempo, lo cual se traduce en pérdidas económicas importantes de la empresa.

Por consiguiente, significa un importante beneficio el hallazgo sobre el efecto que puede causar la restructuración del sistema de gestión de seguridad y salud en el trabajo de la empresa, donde se haya incluido las herramientas para la reducción del ausentismo por casos de hipoacusia en los clientes de la empresa, ya que de esta forma verán, que la empresa toma en cuenta la reducción de lesiones e incidentes que puedan perjudicar los tiempos de entrega de los productos.

\section{CONCLUSIONES}

- En el presente estudio y luego del análisis de sus resultados se determinó evidencia necesaria para aceptar la presencia de una relación que nos permite aseverar que la hipoacusia tiene una influencia significativa en el ausentismo de los trabajadores en la planta MOLICAL S.A.C.

- La investigación nos permite darle una mirada general al ausentismo generado por problemas de hipoacusia en el personal de la planta MOLICAL S.A.C, además la información consignada en la investigación nos servirá como referencia para futuros estudios que busquen determinar la relación entre el ausentismo y otros problemas de salud ocupacional especialmente los problemas de hipoacusia, teniendo en cuenta además que no existen muchas investigaciones al respecto.

- Se determinó que los porcentajes por nivel de hipoacusia en los trabajadores de la planta MOLICAL S.A.C, donde el personal presenta un tipo de hipoacusia moderada y severa son los de mayor porcentaje.

- Se pudo verificar también el porcentaje de ausentismo por parte de los trabajadores de la planta MOLICALS.A.C, presenta un considerable porcentaje de ausencias injustificadas, las misma que se asumen serían causadas por hipoacusia.

\section{AGRADECIMIENTOS}

A mi asesora Dra. Julia Marilú Calderón de Alvarado por sus valiosas recomendaciones e importantes sugerencias, a mi revisor metodológico $\mathrm{Mg}$. Nora Rosa Concepción Malca Casavilca, por sus sugerencias e importante guía y a toda la plana docente de la facultad de Ingeniería Geológica, Minera y Metalúrgica y Geográfica (FIGMMG) de la UNMSM, por el gran apoyo y soporte brindado.

\section{REFERENCIAS}

Allpas Gómez, H. L., Rodriguez Ramos, O., Lezama Rojas, J. L., \& Raraz Vida, O. (2016). Enfermedades del trabajador en una empresa peruana en aplicación de la ley de seguridad y salud en el trabajo. Horizonte Médico (Lima), 16(1), 48-54. https://doi.org/10.24265/horizmed.2016.v16n1.07

Amat, J. (2016). Análisis de Normalidad: gráficos y contrastes de hipótesis. RPubs Estadística Con R, 6. https://www. cienciadedatos.net/documentos/8_analisis_normalidad

Carpio Ayora, M. X., \& Álvarez Pesantez, K. del R. (2017). Estudio Transversal: Hipoacusia Laboral Inducida por Ruido en Personal de Aeronáutica del Ejército Ecuatoriano y Factores Asociados. Quito - Ecuador, 2014 - 2016. Revista Médica Hospital Del José Carrasco Arteaga, 9(2). https:// doi.org/10.14410/2017.9.2.ao.19

OIT. (2010). Lista de enfermedades profesionales de la OIT. Conferencial Internacional Del Trabajo, 1-8. http://www. ilo.org/wcmsp5/groups/public/@ed_protect/@protrav/@ safework/documents/publication/wcms_125164.pdf

Rodríguez García, A. (2014). Análisis del ausentismo laboral por enfermedad en el personal de SEDAPAL, en el periodo 2001-2013. Universidad Nacional Mayor de San Marcos. Facultad de Medicina Humana. Escuela de Post-Grado, Trabajo de Investigación (Especialista en Gestión en Salud). Lima, Perú. https://cybertesis.unmsm.edu.pe/ handle/20.500.12672/13186

Rojas Concha, L. A. (2014). Estudio descriptivo del ausentismo laboral en trabajadores del sistema público de salud en Chile [Universidad de Chile]. http://www.saludpublicachile. cl:8080/dspace/bitstream/handle/123456789/416/Estudio descriptivo del ausentismo laboral en trabajadores del sistema publico de salud en Chile_Leslye Rojas C.pdf?sequence $=1$ 